\title{
Beneficial properties of plants and their derived extracts on the cardiovascular system
}

\author{
Maria Laura Colombo, Chiara Falciola, Stefano Maffè, Franca Davanzo
}

Correspondence to:

Maria Laura Colombo

marialaura.colombo@unito.it

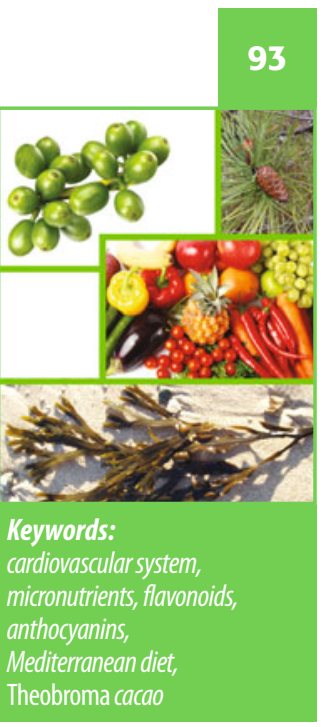

Received: 2 July 2014 / Accepted: 31 July 2014

(C) Springer - CEC Editore 2014

\section{Abstract}

The impact of the quality of our diet on our wellbeing and on the maintenance of our health is now well recognised. In particular, in recent years the importance of micronutrients contained in food has been highlighted. Micronutrients include not only minerals and vitamins (which have long been recognised and studied for their importance), but also a number of other substances contained in small amounts in our food, which are essential for good health. These include glycosides and flavonoids, which may be classified into different chemical classes such as flavanols, anthocyanins, flavones and others. Most of these are typical of the Mediterranean diet. The beneficial properties of the Mediter-

Maria Laura Colombo $(\bowtie)$

Department of Drug and Science Technology

University of Torino

Torino, Italy

Chiara Falciola, Franca Davanzo

Poison Center, Milan

Niguarda Hospital Poison Center

Milano, Italy

Stefano Maffè

Division of Cardiology

SS. Trinità Hospital

Borgomanero

Novara, Italy ranean diet (now recognised by UNESCO as intangible heritage that must be preserved) for human health, and in particular for the heart and the cardiovascular system, may be explained by the content in these compounds. Even wine, an integral part of the Mediterranean diet, when consumed in moderate amounts, has favourable antioxidant activity, improving some aspects of blood circulation. In addition to the intrinsic value of the Mediterranean diet, the recent findings on the beneficial properties of chocolate should be remembered. When consumed in the right quantities, it can also contribute to the proper maintenance of the vascular system.

\section{Introduction}

Almost $60 \%$ of the disease burden in Europe is due to seven leading risk factors: high blood pressure (12.8\%), tobacco (12.3\%), alcohol (10.1\%), high blood cholesterol $(8.7 \%)$, overweight $(7.8 \%)$, low fruit and vegetable intake (4.4\%) and physical inactivity (3.5\%). These risk factors, whose levels are tightly correlated to nutrition patterns, can heavily affect the incidence of cardiovascular disease, which is recognised as the greatest cause of mortality in Europe as well as in the other developed countries [1]. Coronary heart disease, arterio- and atherosclerosis and stroke as well as congestive heart failure also are also the most important causes of perma- 
nent disability [2]. More than 50\% of deaths and disability from cardiovascular disease could be avoided by controlling the major risk factors (hypertension, hypercholesterolaemia, obesity, physical inactivity and tobacco smoke); $80-90 \%$ of people who die from ischaemic heart disease have one or more major risk factors related to lifestyle [3, 4]. In this regard, diet, along with genetic and environmental factors, is considered a major aspect affecting vascular disease outcome as well as longevity [5]. Thus, among non-pharmacological interventions, nutritional recommendations undoubtedly represent a feasible strategy of developing preventive interventions against chronic degenerative diseases. This review will take into consideration positive effects of plants and their extracts on the cardiovascular system and its diseases.

\section{The positive influence of the diet on the cardiovascular system}

Diet is a lifestyle factor that plays a major role in the primary and secondary prevention of numerous chronic diseases, including certain cancers, cardiovascular disease and diabetes. Current dietary recommendations endorse the consumption of a diet rich in fruit and vegetables, since it is now recognised that these foods are healthy for many and varied reasons. The European legislator recommends the use of fiscal measures to promote healthier choices and is working with the agricultural and economic sectors at international, national and local levels to increase the availability of fruits, vegetables and other healthier foods at affordable prices. In order to get the message through to children, the famous '5-a-day fruit and vegetables' information campaign was formulated in European countries [1, 6]. It should also be noted that in these years of economic crisis, the cost of fresh food - mainly vegetables and fruit - can be affected adversely, inducing people to buy cheap food of poor quality. Effective interventions to improve the diet of the population and to improve public health, thereby reducing health-care costs, are therefore needed [7].
In addition to being low in calories and fat and high in fibre, plant-based foods can also contain a number of potentially health-promoting bioactive phytochemicals [8]. Dietary flavonoids have received considerable attention since epidemiological studies suggest that regular consumption of flavonoid-rich foods or beverages is associated with a decreased risk of cardiovascular mortality. The Mediterranean diet (recognised by UNESCO) is rich in these bioactive compounds (resveratrol, flavonoid, anthocyanins, etc.) and it has been clearly demonstrated that it protects against chronic diseases, including coronary heart diseases [9].

The Mediterranean diet is also rich in micronutrients, mainly plant-derived compounds, such as antioxidant vitamins $\mathrm{C}$ and $\mathrm{E}$, which help blood vessels to maintain their flexibility, allowing them to easily dilate and contract in response to proper stimuli [10]. In addition, the powerful antioxidant effect of some glycosides seems to reduce blood pressure by inhibiting inflammatory responses in the blood vessels [11].

It is also worth mentioning that herbal products are generally heterogeneous and can thus produce multiple effects on multiple organ systems (including the cardiovascular system) and, due to their secondary metabolites, may also exert negative effects. For example, some plants such as lily-of-the-valley, oleander, foxglove, broom, monskood, gingko and Ephedra (ma-huang) can have adverse effects on the cardiovascular system. In the case of acute poisoning, the management and treatment are only supportive and symptomatic: there are no antidotes, except for foxglove (digoxin-specific fragments antigen-binding Fab-fragments).

\section{Mediterranean diet}

The positive effects of the diet, and of the Mediterranean diet in particular, on the cardiovascular system are underlined by much evidence from clinical and epidemiological studies and reviews of the evaluation literature $[12,13]$.

The Mediterranean diet (from the Greek $\delta \iota \iota \iota \tau \alpha$, 
originally "way of life, regimen, dwelling") is not simply based on food, but is made up of other aspects such as knowledge, practices, skills and traditions, which range from landscape to table, including crops, harvesting, fishing, preservation, processing, preparation and, particularly, consumption of food. The Mediterranean diet is characterised by a nutritional model that has remained constant over time and space, consisting mainly of olive oil, cereals, fresh or dried fruit and vegetables, a moderate amount of fish, dairy products and meat, and many condiments and spices, all accompanied by wine or infusions. Many scientific studies have been carried out in order to evaluate the effectiveness of the Mediterranean diet, and the discovery of the health-promoting properties of the Mediterranean diet is one of the great successes of epidemiologic research in the second half of the 20th century [14]. The Mediterranean diet is often cited as beneficial for its low content in saturated fat, while monounsaturated fat and dietary fibre content is high. One of the main explanations is thought to be the health effects of olive oil, which is part of the Mediterranean diet.

The Mediterranean diet, in which olive oil is the primary source of fat, is in fact associated with a low mortality caused by cardiovascular disease. We can say that a diet rich in olive oil, or better virgin/extra virgin olive oil, decreases the prothrombotic environment by modifying platelet adhesion, coagulation and fibrinolysis [8]. In recent years olive oil, usually used alone as a condiment or as an ingredient in salad dressing, has been considered, within the Mediterranean diet, as a protective factor against coronary heart disease, a major individual cause of death and morbidity in industrialised countries. In fact, myocardial infarction incidence rates present a high regional variability, with lower rates in European Mediterranean countries than in Northern European ones, the USA and Australia [15]. In 2004, the US Food and Drug Administration announced the availability of a qualified health claim for monounsaturated fat from olive oil which re- duces the risk of coronary heart disease: a qualified health claim on conventional food [16].

Dietary factors explain only part of the health benefits enjoyed by certain Mediterranean cultures. A healthy lifestyle (notably a physically active lifestyle or job) is also beneficial. There is an inverse association between adherence to the Mediterranean diet and the incidence of fatal and non-fatal heart disease in initially healthy middle-aged adults in the Mediterranean region $[17,18]$.

The Mediterranean diet, on the other hand, is high in salt content. Foods such as olives, salt-cured cheeses, anchovies, capers, salted fish roe and salads dressed with olive oil, in fact, may contain high levels of salt.

A 10-year study published in the Journal of the American Medical Association (JAMA) found that adherence to a Mediterranean diet and healthy lifestyle can lower death rates by more than 50\% [19].

The Mediterranean diet advantages are not limited to the cardiovascular system. A mood assessment conducted in the Mediterranean area, as an example, showed that people who followed the Mediterranean diet were less likely to develop depression. In addition, the moderate consumption of red wine improves antioxidant defences, as it contains flavonoids with powerful antioxidant properties $[20,21]$. Interestingly, residents of the Mediterranean area also present very low rates of skin cancer (which is widely believed to be caused by overexposure to solar UV radiation). The incidence of melanomas in the Mediterranean countries is lower than in Northern Europe and significantly lower than in other hot countries such as Australia and New Zealand. It has been hypothesised that some components of the Mediterranean diet may provide protection against skin cancer [22].

\section{Phenolic phytochemicals: flavonoids, anthocyanins, polyphenols}

Phenolic compounds are secondary metabolites mainly found in the plant kingdom. They constitute one of the most abundant groups of natural metabo- 
lites and form an important part of both human and animal diets [23]. These phenolic metabolites' intrinsic function is to protect plants against biological and environmental stresses; in fact, they are mainly synthesised in response to pathogenic attack such as fungal or bacterial infection or high-energy radiation exposure such as prolonged UV exposure. Phenolics, because of their important protective biological functions, are ubiquitous in all plants and therefore are found in almost all vegetable food groups. Common fruits such as apples, cranberries, grapes, raspberries and strawberries and beverages derived from them, such as red wine, apple and orange juices, are rich sources of phenolic compounds. In addition to fruits, vegetables such as cabbage and onion and food grains such as sorghum, millet, barley and peas as well as other legumes are described as important sources of phenolic metabolites. All the natural phenolic molecules are derived from a common biosynthetic pathway, incorporating precursors from the shikimate and/or the acetatemalonate pathways. To increase the solubility and target the phenolics to specific parts of the plant and prevent their enzymatic and chemical degradation, phenolic phytochemicals are often esterified with sugars and other chemical components such as quinic acid through the hydroxyl groups of the phenolic ring.

In recent years, phenolic flavanols, a subgroup of flavonoids, have gained increasing attention as epidemiological investigations have revealed an inverse correlation between the dietary intake of flavanol-containing foods and coronary artery disease mortality [24].

Flavonoids, belonging to the polyphenol family, are mainly water-soluble polyphenolic molecules (except prenylated flavonoids) containing 15 carbon atoms. Their structure consists of two benzene rings linked by a short three-carbon chain (Fig. 1). One of the carbons of the short chain is always connected to a carbon of one of the benzene rings, either directly or through an oxygen bridge, thereby forming a third middle ring, which can be formed by five or six atoms. The flavonoids consist of six major subgroups: chalcone, flavone, flavonol, flavanone, anthocyanins and isoflavonoids. Together with carotenes, flavanoids are also responsible for the colouring of fruits and vegetables, mainly with a yellow-orange colour [25].

In the context of human nutrition, major flavanol sources in the Western diet include tea, grapes and wine, cocoa products, such as chocolate, and various fruits, especially apples and certain berries. Several controlled human dietary intervention studies utilising flavanol-containing foods in concert with nutrient-matched low-flavanol controls have demonstrated a causal relationship between flavanol intake and the modulation of (patho)physiological functions involved in the development and progression of vascular disease. In this context, the attenuation of endothelial dysfunction, decrease in blood pressure, reduction in platelet aggregation and improvement in insulin sensitivity have been observed following clinical dietary interventions with flavanol-containing foods and beverages.

Berries, like many other fruits, are rich in phenolic compounds and these include biphenyls, flavonoids and phenolic acids that help protect cardiac function [26]. They are typically rich in flavonoids and biphenyls such as ellagic acid and represent a large group of secondary plant metabolites. The diversity and complexity of the flavonoids found in berries depend on at least two factors: (i) different variety of aglycones and the high number of glycosides, sometimes in acylated form, and (ii) condensation into complex molecules. Some phenolic acids present in berries are hydroxylated derivatives of benzoic acid and cinnamic acid; other simple phenolics in berries include caffeic, chlorogenic, ferulic, sinapic and $p$-coumaric acids.

A high free radical-scavenging activity of berry extracts toward chemically generated active oxygen species has been described in several studies. In terms of health benefits, increased fruit consumption in daily diets has been shown to significantly reduce the incidence and mortality rates of cancer, cardiovascular disorders and other degenerative diseases 
<smiles>[R]c1cc(-c2oc3cc(O)cc(O)c3c(=O)c2O)cc([R])c1[R]</smiles>

Flavonols<smiles>[R]c1cc(-c2cc(=O)c3c(O)cc(O)cc3o2)cc([R])c1[R]</smiles>

Flavonones

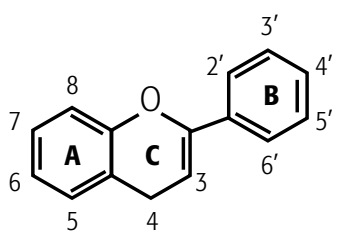

Basic flavonoid structure<smiles>[R]c1cc(-c2cc(=O)c3c(O)cc(O)cc3o2)cc([R])c1[R]</smiles>

Flavones<smiles>[R]c1cc(-c2oc3cc(O)cc(O)c3c(=O)c2O)cc([R])c1O</smiles>

Anthycyanins<smiles>[R]c1cc(-c2oc3cc(O)cc(O)c3c(=O)c2O)cc([R])c1[R]</smiles>

Flavanols

Figure 1 Chemical structures of different classes of flavonoid (from [25])

caused by oxidative stress. Flavonoids would be able to act on multiple cellular targets, in micromolar doses. Moreover, while a drug acts in a specific way, a natural compound acts in a modular manner by interacting with several cell sites [27]. Phenolics are ubiquitous in plants, but seeds and skins are especially rich sources of phenolics, probably because of the role in protecting the fruit and the seed to ensure safe propagation of the species. However, several phenolics exist in conjugated forms either with sugars (primarily glucose) as glycosides or other moieties. This conjugation occurs via the hydroxyl groups of the phenolics, which reduces their ability to function as good antioxidants since availability of free hydroxyl groups on the phenolic rings is important for resonance stabilisation of free radicals.
Lowered antioxidant capacity has direct implications on decreasing functionalities for health benefit when these phenolics are ingested via food [28]. Therefore, if free phenolics are released from their glycosides or other conjugates, then the antioxidant and thus their function for health benefit could be enhanced. Enzymatic hydrolysis of these phenolic glycosides appears to be an attractive means of increasing the concentration of free phenolic acids in fruit juice and wines to enrich taste, flavour and aroma.

Anthocyanins are another interesting group of polyphenol occurring pigments responsible for the red-blue-violet colour, according to the $\mathrm{pH}$ value of the cell sap of many fruits and vegetables. Anthocyanins are water-soluble pigments, not found in the plastids like other pigments, but in the vac- 
uoles of plant cells. There are over 300 structurally different anthocyanins that have been identified in nature [29]. Numerous studies indicate the potential effect that this family of compounds may have in reducing the incidence of cardiovascular diseases, cancer, hyperlipidaemias and other chronic diseases through the intake of anthocyanin-rich foods [30]. Commercial anthocyanin colorants are mostly derived from fruits and vegetables. These sources include red grape, elderberry, blackcurrant, blackberry, raspberry, black chokeberry, red cabbage, purple carrot, purple corn, red radish and purple sweet potato. The largest natural commercial source for anthocyanins is red grape skin, followed by elderberry, purple carrot and red cabbage; in particular the anthocyanins from purple carrot juice have good stability and high antioxidant activity [31].

\section{Theobroma cacao polyphenols}

In the past 15 years there has been increased interest in the potential health-related benefits of antioxidants and phytochemicals of dark chocolate and cocoa, which have been intensively studied in recent years mostly driven by the large content of flavanols and related compounds in cocoa beans [32]. Cacao bean, the seed of Theobroma cacao, is known to be rich in polyphenols, such as the procyanidin monomers ((+)-catechin and (-)-epicatechin) and oligomers (B-type procyanidins that are linked by $\mathrm{C} 4-\mathrm{C} 8$ bonds). Hundreds of studies have reported the content in bioactive compounds, the chemical compositions and the health benefits of cocoa and cocoa-derived products [33]. Since cocoa products have greater antioxidant capacity and greater amounts of flavonoids per serving than all teas and red wines, it is important to underline the potential effects of chocolate on cardiovascular disease. Since ancient times, chocolate has been used as a medicinal remedy and has been proposed in medicine today for preventing various chronic diseases. While chocolate has also sometimes been criticised for its saturated fat content, mostly in the form of long-chain stearic acid, dark chocolate has also been praised for its antioxidant potential. A full range of health benefits can today be associated to the actions of flavanols and procyanidins on vascular function. These benefits are mainly ascribed to diets rich in flavanols and procyanidins. Chocolate and cocoa derivatives are among the most valuable components of such a diet [34].

A growing number of clinical studies indicate that regular ingestion of $T$. cacao flavanol exerts a range of effects potentially favourable to vascular healthimproving endothelial function, reducing elevated blood pressure, increasing insulin sensitivity and suppressing platelet aggregation. There is recent evidence that the epicatechin content of cocoa is primarily responsible for its favourable impact on vascular endothelium, which reflects both an acute and chronic up-regulation of nitric oxide production. As is well known, physiological levels of nitric oxide support vascular health and efficient tissue perfusion by promoting vasodilation, opposing inflammation and structural remodelling in the vascular wall, and stabilising platelets. A European Food Safety Authority (EFSA) Scientific Opinion concludes that a cause and effect relationship between the consumption of cocoa flavanols and maintenance of normal endothelium-dependent vasodilation has been established. The following wording reflects the scientific evidence: "Cocoa flavanols help maintain endothelium-dependent vasodilation, which contributes to normal blood flow". In order to obtain the claimed effect, $200 \mathrm{mg}$ of cocoa flavanols should be consumed daily. This amount could be provided by 2.5 $\mathrm{g}$ of high-flavanol cocoa powder or $10 \mathrm{~g}$ of high-flavanol dark chocolate, both of which can be consumed in the context of a balanced diet. The target population is the general population [35].

On the basis of the above data, flavanol-rich chocolate and cocoa products have attracted interest as non-pharmacological treatment for high blood pressure, a known risk factor for cardiovascular disease: even small reductions in blood pressure substantially reduce cardiovascular risk. Polyphenols, 
in particular flavanols in cocoa products, have been shown to increase the formation of endothelial nitric oxide, which promotes vasodilation and consequently may lower blood pressure.

Some evidence identified an array of potential mechanisms through which chocolate and cocoa products may promote cardiovascular health and provide cardioprotective effects. Specifically, chocolate and cocoa-related products have been shown to decrease or inhibit both LDL oxidation and platelet activation or function, to enhance serum lipid profiles, to favourably modify eicosanoid synthesis, to lower blood pressure, to promote endothelium-dependent relaxation or dilation and to inhibit free radical-induced erythrocyte haemolysis [33, 36]. Furthermore, preliminary in vitro investigations have suggested that cocoa flavanols or procyanidins may possess immunoregulatory effects and may help to modulate immune responses. In addition, several reports have indicated that the susceptibility of low-density lipoprotein (LDL) to oxidation was significantly decreased in humans and high cholesterolaemic rabbits by dietary supplementation with cocoa or its procyanidin fraction. Moreover, many reports support the possibility that procyanidins in cacao can prevent cardiovascular disease by improving blood flow rate, improving platelet function, changing inflammatory responses in endothelial cells of blood vessels and so on. On the other hand, many reports attribute the decrease in plasma cholesterol levels in experimental animals to the ingestion of chocolate, tea, citrus fruit, grape and wine flavanoids $[37,38]$.

\section{Conclusions}

From all of these observations, it is clear that the value of food is no longer considered only its nutrient content (and thus its nutritional power), but also its role as an irreplaceable source of valuable molecules for our well-being. These molecules are also called "micronutrients" and their presence in our daily diet is increasingly valued. These micronutrients are mostly of vegetable origin and the
Mediterranean diet is particularly rich in them. Some sources with high amounts of micronutrients important for human health are extra virgin olive oil, yellow-, red- and purple-coloured fruits and vegetables, nuts and cocoa $[39,40]$. In particular, micronutrients exert a beneficial effect on the heart and on the cardiovascular system as a whole. It should also be noted that cardiovascular diseases are a major cause of death, especially in industrialised countries. Thus, the adoption of a healthy and balanced diet with regular consumption of fruits and vegetables can improve our general health and reduce health-care costs.

\section{Human and Animal Rights}

All procedures followed were in accordance with the ethical standards of the responsible committee on human experimentation (institutional and national) and with the Helsinki Declaration of 1975, as revised in 2000(5). Informed consent was obtained from all patients for being included in the study.

\section{Conflict of interest}

Maria Laura Colombo, Chiara Falciola, Stefano Maffè and Franca Davanzo declare that they have no conflict of interest.

\section{References}

1. WHO Regional Office for Europe (2007) Gaining health: The European Strategy for the Prevention and Control of Noncommunicable Diseases. Denmark, ISBN 92-890 21799

2. Morgan Griffin R. Leading causes of disability. WebMD. http://www.webmd.com/healthy-aging/features/top-causesdisability (accessed 30 June 2014)

3. WHO (2003) The World Health Report. WHO

4. WHO (2013) The World Health Report. WHO

5. Trichopoulou A, Vasilopoulou E (2000) Mediterranean diet and longevity. Br J Nutr 84[Suppl 2]:S205-209

6. John JH, Ziebland S, Yudkin P, Roe LS, Neil H (2002) Effects of fruit and vegetable consumption on plasma antioxidant concentrations and blood pressure: a randomised controlled trial. Lancet 359:1969-1974

7. Ball K, McNaughton SA, Mhurchu CN, Andrianopoulos N, Inglis V, McNeilly B, Le H, Leslie D, Pollard C, Crawford D (2011) Supermarket Healthy Eating for Life (SHELf): protocol of a randomised controlled trial promoting healthy food and beverage consumption through price reduction and skill-building strategies. BMC Public Health 11:715

8. Joint WHO/FAO Expert Consultation on Diet (2003) Nu- 
trition and the Prevention of Chronic Disease. WHO Technical Report Series $N^{\circ} 916$

9. Pandey KB, Rizvi SI (2009) Plant polyphenols as dietary antioxidants in human health and disease. Oxid Med Cell Longev 2(5):270-278

10. WHO. Micronutrients. http://www.who.int/nutrition/topics/micronutrients/en/ (accessed 30 June 2014)

11. Grossman E (2008) Does increased oxidative stress cause hypertension? Diabetes Care 31[Suppl 2]:S185-189

12. Charles RL, Rudyk O, Prysyazhna O, Kamynina A, Yang J, Morisseau C, Hammock BD, Freeman BA, Eaton P (2014) Protection from hypertension in mice by the Mediterranean diet is mediated by nitro fatty acid inhibition of soluble epoxide hydrolase. Proc Natl Acad Sci USA doi: 10.1073/pnas. 1402965111

13. British Heart Foundation. Five top tips for making your meals more Mediterranean. http://www.bhf.org.uk/heart\%20matters\%20online/january\%20february\%202014/nutrition/medi terranean\%20diet.aspx (accessed 30 June 2014)

14. Serra-Majem L, Roman B, Estruch R (2006) Scientific evidence of interventions using the Mediterranean diet: a systematic review. Nutr Rev 64:S27-47

15. The Fifth Joint Task Force of the European Society of Cardiology and Other Societies on Cardiovascular Disease Prevention in Clinical Practice (2012) European Guidelines on cardiovascular disease prevention in clinical practice. Eur J Prev Cardiol 19:585-667

16. FDA (2004) FDA allows qualified health claim to decrease risk of coronary heart disease. http://www.fda.gov/newsevents/newsroom/pressannouncements/2004/ucm108368.ht m (accessed 30 June 2014)

17. Panagiotakos DB, Pitsavos C, Stefanadis C (2006) Dietary patterns: a Mediterranean diet score and its relation to clinical and biological markers of cardiovascular disease risk. Nutr Metab Cardiovasc Dis 16:559-568

18. Urpi-Sarda M, Casas R, Chiva-Blanch G, Romero-Mamani ES, Valderas-Martínez P, Arranz S, Andres-Lacueva C, Llorach R, Medina-Remón A, Lamuela-Raventos RM, Estruch $R$ (2012) Virgin olive oil and nuts as key foods of the Mediterranean diet effects on inflammatory biomarkers related to atherosclerosis. Pharm Res 65:577-583

19. Knoops KT, de Groot LC, Kromhout D, Perrin AE, MoreirasVarela O, Menotti A, van Staveren WA (2004) Mediterranean diet, lifestyle factors, and 10-year mortality in elderly European men and women: the HALE project. JAMA 292:14331439

20. Kushi LH, Lenart EB, Willett WC (1995) Health implications of Mediterranean diets in light of contemporary knowledge. 2. Meat, wine, fats, and oils. Am J Clin Nutr 61[Suppl]:1416S$1427 \mathrm{~S}$

21. Di Renzo L, Carraro A, Valente R, Iacopino L, Colica C, De Lorenzo A (2014) Intake of red wine in different meals modulates oxidized LDL level, oxidative and inflammatory gene expression in healthy people: a randomized crossover trial. Oxid Med Cell Longev 2014:681318

22. Fortes C, Mastroeni S, Melchi F, Pilla MA, Antonelli G, Camaioni D, Alotto M, Pasquini P (2008) A protective effect of the Mediterranean diet for cutaneous melanoma. Intern J Epidemiol 37:1018-1029

23. Cheynier V (2012) Phenolic compounds: from plants to foods. Phytochem Res 11:153-177

24. Heiss C, Keen CL, Kelm M (2010) Flavanols and cardiovascular disease prevention. Eur Heart J 31:2583-92

25. Kumar S, Pandey AK (2013) Chemistry and biological activities of flavonoids: an overview. Sci World J 2013:162750

26. McCullough ML, Peterson JJ, Patel R, Jacques PF, Shah R, Dwyer JT (2012) Flavonoid intake and cardiovascular disease mortality in a prospective cohort of US adults. Am J Clin Nutr 95:454-464

27. Arango D, Morohashi K, Yilmaz A, Kuramochi K, Parihar A, Brahimaj B, Grotewold E, Doseff AI (2013) Molecular basis for the action of a dietary flavonoid revealed by the comprehensive identification of apigenin human targets. Proc Natl Acad Sci USA 110:E2153-2162

28. Carey FA (2001) Organic chemistry. McGraw-Hill, Boston

29. Castañeda-Ovando A, Pacheco-Hernández M de L, PáezHernández ME, Rodríguez JA, Galán-Vidal CA (2009) Chemical studies of anthocyanins: a review. Food Chem 113:859871

30. Cassidy A, Mukamal KJ, Liu L, Franz M, Eliassen AH, Rimm EB (2013) High anthocyanin intake is associated with a reduced risk of myocardial infarction in young and middleaged women. Circulation 127:188-196

31. Poudyal H, Panchal S, Brown L (2010) Comparison of purple carrot juice and b-carotene in a high-carbohydrate, high-fat diet-fed rat model of the metabolic syndrome. Br J Nutr 104:1322-1332

32. Carrillo LC, Londoño-Londoño J, Gil A (2014) Comparison of polyphenol, methylxanthines and antioxidant activity in Theobroma cacao beans from different cocoa-growing areas in Colombia. Food Res Int 60:273-280

33. Paoletti R, Conti A, Visioli F, Poli A (2001) Chocolate and health. Springer, Milan

34. Osakabe N (2013) Flavan-3-ols improve metabolic syndrome risk factors: evidence and mechanisms. J Clin Biochem Nutr 52:186-192

35. EFSA Panel NDA (2012) Scientific opinion on the substantiation of a health claim related to cocoa flavanols and maintenance of normal endothelium-dependent vasodilation pursuant to Article 13(5) of Regulation (EC) No 1924/2006. EFSA J 10:2809-2830

36. Conti A, Pinorini-Godly MT, Colombo ML (2013) Cocoa and chocolate in humans: the rationale for clinical studies on healthy volunteers. In: Watson RR et al (eds) Chocolate in health and nutrition, Nutrition and Health 7, Springer Science and Business Media, New York 
37. Murphy KJ, Chronopoulos AK, Singh I, Francis MA, Moriarty H, Pike MJ, Turner HA, Mann NJ, Sinclair AJ (2003) Dietary flavanols and procyanidin oligomers from cocoa (Theobroma cacao) inhibit platelet function. Am J Clin Nutr 77:1466-1473

38. Georgiev V, Ananga A, Tsolova V (2014) Recent advances and uses of grape flavonoids as nutraceuticals. Nutrients 6:391-415
39. Ames BN (2006) Low micronutrient intake may accelerate the degenerative diseases of aging through allocation of scarce micronutrients by triage. Proc Natl Acad Sci USA 103:17589-17594

40. University of York. Botany and health: very small chemical changes to dietary flavonoids cause very large effects on human immune system. ScienceDaily 30 August. www.sciencedaily.com/releases/2013/08/130830091800.htm 